\title{
The Impact of Tidal Disruption Events on Galactic Habitability
}

\author{
E. Pacetti ${ }^{1}$, A. Balbi ${ }^{1 \star}$, M. Lingam ${ }^{2,3}$, F. Tombesi ${ }^{1,4,5,6}$ and E. Perlman ${ }^{2}$ \\ ${ }^{1}$ Department of Physics, University of Rome "Tor Vergata", Via della Ricerca Scientifica 1, I-00133 Rome, Italy \\ ${ }^{2}$ Department of Aerospace, Physics and Space Sciences, Florida Institute of Technology, Melbourne FL 32901, USA \\ ${ }^{3}$ Institute for Theory and Computation, Harvard University, Cambridge MA 02138, USA \\ ${ }^{4}$ INAF Astronomical Observatory of Rome, Via Frascati 33, I-00078 Monte Porzio Catone, Italy \\ ${ }^{5}$ Department of Astronomy, University of Maryland, College Park, MD 20742, USA \\ ${ }^{6}$ NASA - Goddard Space Flight Center, Code 662, Greenbelt, MD 20771, USA
}

Accepted XXX. Received YYY; in original form ZZZ

\begin{abstract}
Tidal Disruption Events (TDEs) are characterized by the emission of a short burst of highenergy radiation. We analyze the cumulative impact of TDEs on galactic habitability using the Milky Way as a proxy. We show that X-rays and extreme ultraviolet (XUV) radiation emitted during TDEs can cause hydrodynamic escape and instigate biological damage. By taking the appropriate variables into consideration, such as the efficiency of atmospheric escape and distance from the Galactic center, we demonstrate that the impact of TDEs on galactic habitability is comparable to that of Active Galactic Nuclei. In particular, we show that planets within distances of $\sim 0.1-1 \mathrm{kpc}$ could lose Earth-like atmospheres over the age of the Earth, and that some of them might be subject to biological damage once every $\gtrsim 10^{4} \mathrm{yrs}$. We conclude by highlighting potential ramifications of TDEs and argue that they should be factored into future analyses of inner galactic habitability.
\end{abstract}

Key words: astrobiology, planets and satellites: atmospheres, planets and satellites: terrestrial planets, quasars: supermassive black holes, transients: tidal disruption events, Galaxy: nucleus

\section{INTRODUCTION}

The concept of habitability is complex due to its inherently multifaceted nature. In particular, habitability is regulated by a plethora of processes spanning a diverse range of spatial and temporal scales. While most studies have exclusively focused on habitability at the planetary or stellar levels (Cockell et al. 2016; Lingam \& Loeb 2019b), it has been increasingly appreciated over the past couple of decades that high-energy astrophysical phenomena may regulate habitability on galactic scales (Gonzalez 2005; Prantzos 2008). Most studies in this realm have tended to focus on the effects of supernovae and Gamma Ray Bursts; see the recent reviews by Gowanlock \& Morrison (2018); Kaib (2018).

Over the past few years, the impact of Active Galactic Nuclei (AGNs) has attracted greater attention, building on earlier studies (Clarke 1981; Gonzalez 2005). In particular, recent studies indicate that AGNs could drive the complete depletion of Earth-like atmospheres across a significant fraction of planets in galaxies (Balbi \& Tombesi 2017; Forbes \& Loeb 2018; Wisłocka et al. 2019), promote disruption of biospheres due to elevated radiation fluxes (Balbi \& Tombesi 2017; Lingam et al. 2019; Amaro-Seoane \& Chen 2019), and permit the synthesis of prebiotic compounds and carbon fixation (Lingam et al. 2019; Liu et al. 2020). A crucial property of AGNs

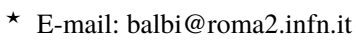

should, however, be borne in mind: they are relatively short-lived, and only a small fraction of all galaxies are "active" at any given moment in time (Krolik 1999).

Aside from AGNs, another crucial high-energy process intrinsically associated with supermassive black holes (SMBHs) is tidal disruption events (TDEs). ${ }^{1}$ The existence of TDEs was predicted and modeled in the 1970s and 1980s (Hills 1975; Frank \& Rees 1976; Lacy et al. 1982). These phenomena arise when stars traverse too close to SMBHs, and are consequently disrupted by the latter's tidal field (Komossa 2015; Alexander 2017; French et al. 2020). Despite the fact that TDEs are expected to recur in a wide range of galaxies, primarily those which host SMBHs of masses $\sim 10^{6}-10^{8} M_{\odot}$ (Stone et al. 2019), there have been no studies devoted to assessing their impact on galactic habitability.

Hence, our goal is to explore the cumulative impact of TDEs by specifically focusing on the Milky Way and Sagittarius A* (Sgr $\mathrm{A}^{*}$ ) herein. The outline of this paper is as follows. In Sec. 2, we describe some basic properties of TDEs and our methodology. We present the ensuing results in Sec. 3, and analyze the ramifications for habitability in Sec. 4.

\footnotetext{
1 Although we will treat TDEs and AGNs as independent phenomena hereafter, Padmanabhan \& Loeb (2020) recently suggested that the former might trigger the latter in some instances, especially at higher redshifts.
} 


\section{Pacetti et al.}

\section{MODELING THE IMPACT OF TDES ON HABITABILITY}

In this Section, we describe our methodology to quantify the effects on habitability engendered by TDEs. Stars with mass $M_{\star}$ and radius $R_{\star}$ undergo tidal disruption if they approach the SMBH of mass $M_{B H}$ closer than the tidal radius $R_{t} \approx R_{\star}\left(M_{B H} / M_{\star}\right)^{1 / 3}$ (Hills 1975 ). During the course of a TDE, roughly one-half of the stellar mass escapes on a hyperbolic orbit, whereas the rest falls inward onto the black hole at a rate $\dot{M}$. The accretion rate is often modeled via the following power-law parametrization (Rees 1988; Lodato et al. 2015):

$\dot{M}=\frac{M_{\star}}{3 t_{\min }}\left(\frac{t+t_{\min }}{t_{\min }}\right)^{-5 / 3}$,

where $t_{\min }$ is defined as

$t_{\text {min }}=\frac{\pi}{\sqrt{2}}\left(\frac{R_{t}}{R_{\star}}\right)^{3 / 2} \sqrt{\frac{R_{t}^{3}}{G M_{B H}}} \approx 41 M_{6}^{1 / 2} \mathrm{~d}$,

for a solar-type star, with $M_{6}=M_{B H} /\left(10^{6} M_{\odot}\right)$. The power-law exponent of $-5 / 3$ in (1) is not accurate sensu stricto (Auchettl et al. 2017), but it suffices for our purposes. The energy acquired during accretion is re-emitted by the accretion disk over the span of $\sim 1$ yr (Komossa 2015) with a luminosity $L=\eta \dot{M} c^{2}$, where $\eta \sim 0.1$ is the typical radiative efficiency. By employing (1) in conjunction with the definition of $L$, the upper bound on the total energy $\left(E_{t o t}\right)$ emitted is given by

$E_{\text {tot }}=\int_{0}^{\infty} L d t=\frac{\eta M_{\star} c^{2}}{2}$,

where the "clock" is started at $t=0$ hereafter. In order to estimate the fraction $f_{X U V}$ of total energy emitted in the X-ray and extreme ultraviolet (XUV) band, we opt to model the TDE as a black body with a temperature of $k_{B} T_{\star} \approx 100 \mathrm{eV}$ (Saxton et al. 2017), and specify the XUV wavelength range of $1.24 \times 10^{-3}<\lambda<1.24 \times 10^{2}$ $\mathrm{nm}$, i.e., corresponding to energies of $10-10^{6} \mathrm{eV}$. For the black body ansatz, we find that $f_{X U V} \approx 0.99$, implying that the overwhelming majority of the total energy is emitted as XUV photons.

\subsection{Hydrodynamic loss}

The flux of XUV photons $\left(F_{X U V}\right)$ at a given distance $D$ is

$F_{X U V}=\frac{f_{X U V} L e^{-\tau}}{4 \pi D^{2}}$

under the assumption of isotropic radiation; note that $\tau$ denotes the effective optical depth along the line of sight that is discussed further below. The XUV incident on a planet at this distance is capable of driving atmospheric escape through a number of channels (Owen 2019; Lingam \& Loeb 2019b). We will model the resultant atmospheric loss via energy-limited hydrodynamic escape (Balbi \& Tombesi 2017), thereby yielding

$M_{\text {lost }}=\frac{3}{4} \frac{\varepsilon}{G \rho_{p}} \int_{0}^{\infty} F_{X U V} d t$,

where $M_{\text {lost }}$ is the mass lost during a single TDE, $\rho_{p}$ is the density of the planet, and $0.1 \leq \varepsilon \leq 0.6$ is the heating efficiency that describes the conversion of incident power into atmospheric loss. By combining (3), (4) and (5), we obtain

$M_{\text {lost }}=\frac{3}{4} \frac{\varepsilon}{G \rho_{p}} \frac{f_{X U V} e^{-\tau}}{4 \pi D^{2}}\left(\frac{\eta M_{\star} c^{2}}{2}\right)$.
There are two important caveats concerning our model. First, when it comes to $\tau$, there are two contributors: (i) the dusty torus at the center of an active galaxy (Urry \& Padovani 1995), and (ii) the interstellar medium. While we take (i) into account along the lines described in Balbi \& Tombesi (2017), tackling (ii) is much more complex as it depends not only on the wavelength range but also $D$ and the galactic morphology (e.g., spiral or elliptical). Hence, as with prior publications on AGNs, we do not incorporate this contribution to $\tau$. The second limitation stems from the exclusion of the beaming effect in TDEs (Dai et al. 2018; French et al. 2020). Assessing the impact of beamed emission is rendered difficult, because the number of stars affected by the beamed emission depends not only on the solid angle $\Omega$ but also the orientation of the beam axis and the galactic morphology. For this reason, we restrict ourselves to studying isotropic emission hereafter.

\subsection{Biological hazards}

A proper assessment of the biological hazards entails more subtleties because the characteristics of putative biota are unknown, as are the properties of the planetary atmosphere under question.

On account of these reasons, we will utilize a simplified approach to assess the impact of XUV radiation on biota. It is known that ionizing radiation becomes lethal to Earth-based organisms after a certain critical threshold is exceeded (Dartnell 2011). As laboratory experiments are performed over a finite time, the thresholds are typically expressed in units of fluences. We adopt critical fluences of $\mathcal{F}_{c} \approx 5 \times 10^{5} \mathrm{erg} \mathrm{cm}^{-2}$ for eukaryotic multicellular lifeforms and $\mathcal{F}_{c} \approx 5 \times 10^{7} \mathrm{erg} \mathrm{cm}^{-2}$ for prokaryotic microbes, based on Scalo \& Wheeler (2002, Section 6.2) and Balbi \& Tombesi (2017).

The electromagnetic energy emitted in the XUV band during a single TDE $(E)$ is found by integrating the luminosity over the characteristic timescale of $\Delta t \sim 1 \mathrm{yr}$, analogous to estimating $E_{t o t}$ in (3), thus yielding

$E=\int_{0}^{\Delta t} f_{X U V} \cdot L d t$

For a solar-type star that undergoes tidal disruption, the above formula yields $E \approx 7 \times 10^{52} \mathrm{erg}$, which is consistent with prior theoretical estimates (e.g., Lu \& Kumar 2018). Under the assumption of isotropic emission, the fluence $\mathcal{F}$ at distance $D$ is given by

$\mathcal{F}=\frac{E e^{-\tau}}{4 \pi D^{2}}=5.8 \times 10^{8} \mathrm{erg} \mathrm{cm}^{-2} e^{-\tau}\left(\frac{D}{1 \mathrm{kpc}}\right)^{-2}$.

In comparison, the high-energy electromagnetic radiation delivered by a supernova over the same timescale of $\sim 1 \mathrm{yr}$ at distance $D$ is roughly predicted to be (Ellis \& Schramm 1995, Equation 1):

$\mathcal{F}=6.6 \times 10^{7} \mathrm{erg} \mathrm{cm}^{-2}\left(\frac{D}{1 \mathrm{pc}}\right)^{-2}$.

When the fluence becomes equal to the critical values delineated previously, the corresponding distances $\left(D_{c}\right)$ are estimated using

$D_{c}=\sqrt{\frac{E e^{-\tau}}{4 \pi \mathcal{F}_{c}}}$.

There are two caveats worth mentioning at this juncture. First, the threshold has been expressed in terms of fluence, but the timescale over which a given fluence is exceeded also plays a vital role. $^{2}$ Over the span of $\sim 1 \mathrm{yr}$ considered above, the XUV fluence

2 To put it differently, even a small flux will eventually exceed $\mathcal{F}_{c}$ after sufficient time. 
of the modern Sun is $\mathcal{F}_{\oplus} \approx 1.5 \times 10^{8} \mathrm{erg} \mathrm{cm}^{-2}$, based on the XUV flux of $F_{\oplus} \approx 4.64 \mathrm{erg} \mathrm{cm}^{-2} \mathrm{~s}^{-1}$ (Ribas et al. 2005). Hence, the XUV fluence of a Sun-like star is actually higher than (8) in the event that $D \gtrsim 1 \mathrm{kpc}$. Second, the majority of XUV radiation will be absorbed by a thick atmosphere (Scalo \& Wheeler 2002; Melott \& Thomas 2011), implying that the above results are accurate only for highly tenuous atmospheres, and should therefore be viewed as upper bounds. However, regardless of the atmosphere, what is undeniable is that the top-of-atmosphere XUV fluence at $D \sim 0.1 \mathrm{kpc}$ is orders of magnitude higher than $\mathcal{F}_{\oplus}$ as well as a supernova at $\sim 1$ pc, as seen from (8) and (9). In other words, the effects induced by TDEs at this distance would be comparable or higher than $\mathrm{SNe}$ at $\sim 1 \mathrm{pc}$, which are expected to be considerable (Gehrels et al. 2003; Branch \& Wheeler 2017).

Hence, a different approach entails the adoption of (i) flux instead of fluence, and (ii) photon wavelengths that can penetrate through an Earth-like atmosphere. To this end, we adopt the methodology proposed in Lingam et al. (2019), that was based on Melott \& Thomas (2011). The basic idea is that doubling the top-ofatmosphere (TOA) UV-C flux, for a world akin to Hadean-Archean Earth, might result in large-scale extinction of biota. The flux in this wavelength range is determined via

$F_{U V C}=\frac{f_{U V C} L e^{-\tau}}{4 \pi D^{2}}$,

where $f_{U V C}$ is the energy fraction of radiation emitted at UVC wavelengths. By employing the black body spectrum with $T_{\star}$, we obtain $f_{U V C}$. By equating $F_{U V C}$ with the TOA UV-C flux of $2.2 \times 10^{3} \mathrm{erg} \mathrm{cm}^{-2} \mathrm{~s}^{-1}$ (Rugheimer et al. 2015), we determine the value of $D$ at which disruption of the biosphere may be possible. We caution that, even when it comes to such worlds, it is well-known that $\lesssim 1 \mathrm{~m}$ of water can effectively protect aquatic organisms from UV photons (Cleaves \& Miller 1998); similar shielding could arise from soils and UV screening compounds (Cockell \& Knowland 1999). Furthermore, terrestrial worlds with massive atmospheres (e.g., Super-Earths) or substantial haze densities are capable of conferring additional protection against XUV radiation (Arney et al. 2016; Lingam \& Loeb 2019b). It is, therefore, important to recognize that our estimates are partly heuristic.

\section{RESULTS}

We apply the methodology outlined in the previous section and adumbrate the salient results.

\subsection{Effects on the atmosphere}

As one may expect, due to their transient nature, a single TDE does not cause significant atmospheric loss. A terrestrial planet at $D=0.1 \mathrm{kpc}$ is depleted of merely $\sim 10^{-5}$ of the total mass of Earth's present-day atmosphere (denoted by $M_{\text {atm }, \oplus} \approx 5.1 \times 10^{21}$ g) for $\varepsilon=0.6$ during a single TDE. Therefore, it is more instructive to calculate the cumulative atmospheric escape due to TDEs over a span of $5 \mathrm{Gyr}$; this timescale is chosen as it corresponds to the age of our Solar system. We adopt a TDE rate of $\sim 10^{-4} \mathrm{yr}^{-1}$ per galaxy (Komossa 2015). The results are depicted in Figure 1.

It is important to recognize that the atmospheric loss from TDEs complements the depletion caused by the AGN phase. Hence, it is necessary to gauge the relative degree of atmospheric loss caused by TDEs and the AGN phase. In Figure 2, we show the atmospheric mass resulting from TDEs and the AGN phase. For

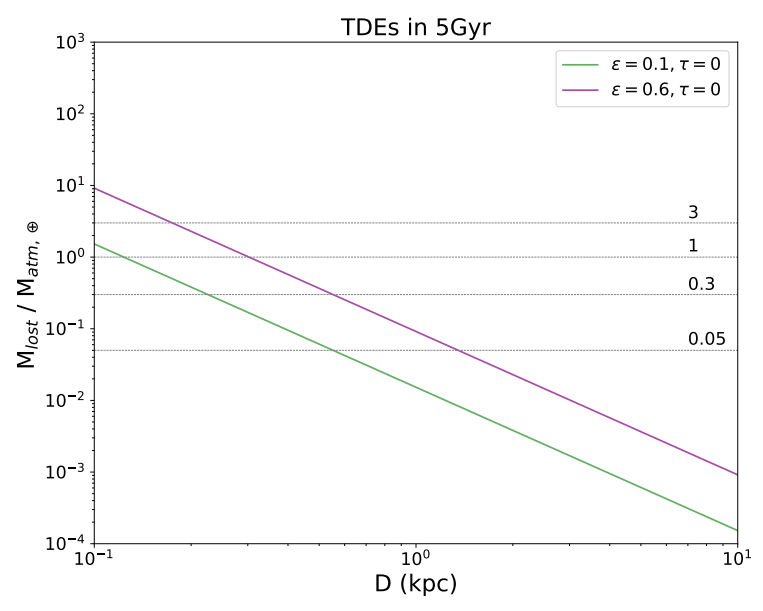

Figure 1. Total mass (in units of Earth's present-day atmospheric mass) lost by a terrestrial planet at distance $D$ due to TDEs over a span of 5 Gyr. We have adopted a TDE rate of $\sim 10^{-4} \mathrm{yr}^{-1}$ per galaxy. The results are shown for two values of the efficiency of hydrodynamic escape $(\varepsilon)$. In both cases, we consider isotropic radiation propagating in an optically thin medium.

fixed values of the free parameters, namely $D, \varepsilon$ and $\tau$, we observe that the mass loss due to TDEs is a few times smaller than the AGN phase. However, it is important to recall that many TDEs exhibit beamed emission (Dai et al. 2018). When the beaming effect is included, the mass loss might become comparable to (or even higher than) the AGN phase, although uncertainties remain (see Sec. 2.1). On the other hand, the number of worlds that would be affected by beamed emission are correspondingly fewer than the isotropic case.

It is not easy to extract a generic result regarding the extent of the zone where atmospheric loss due to TDEs is prominent. The reason stems from the fact that there are three variables involved, namely, $\varepsilon, \tau$ and $\Omega$. Nonetheless, in all cases, we find that $M_{\text {lost }} \gtrsim M_{\text {atm }, \oplus \text { is fulfilled when the distance obeys } D \lesssim 0.1-1}$ $\mathrm{kpc}$. In contrast, if we are interested in the zone where Mars-like atmospheres are completely lost, it is more than an order of magnitude higher, i.e., the critical distance becomes $\sim 1-10 \mathrm{kpc}$. On the other hand, the depletion of massive Super-Earth atmospheres is much more difficult, and thus shrinks the zone to $\sim 10 \mathrm{pc}$.

Before moving ahead, a few caveats must be spelt out. The formalism in Sec. 2.1 does not account for other source and sink terms (Catling \& Kasting 2017), such as volcanic outgassing, continental weathering, and biological processes (e.g., photosynthesis). In other words, we assumed that the atmosphere exists in steadystate, with the sole exception of transient high-energy astrophysical processes that subsequently drive the intermittent atmospheric loss. Second, we invoked the paradigm of energy-limited hydrodynamic escape, but magnetohydrodynamic escape mechanisms are potentially dominant for certain inner Solar system worlds and exoplanets (Brain et al. 2016; Dong et al. 2017, 2018).

Lastly, it is possible that the planet's atmosphere may return to its "normal" levels in the $\gtrsim 10^{4}$ yrs separating TDEs (Stone et al. 2020). However, the loss of $10^{-5}$ of its atmosphere in a short timespan of $1 \mathrm{yr}$ at the distance of $\sim 0.1 \mathrm{kpc}$ as noted earlier could nevertheless be detrimental. In particular, the escape rate becomes higher than its background value due to stellar irradiation by several orders of magnitude at $\sim 0.1 \mathrm{kpc}$. Such a rapid increase has been posited as one of the chief reasons for the Triassic-Jurassic mass 

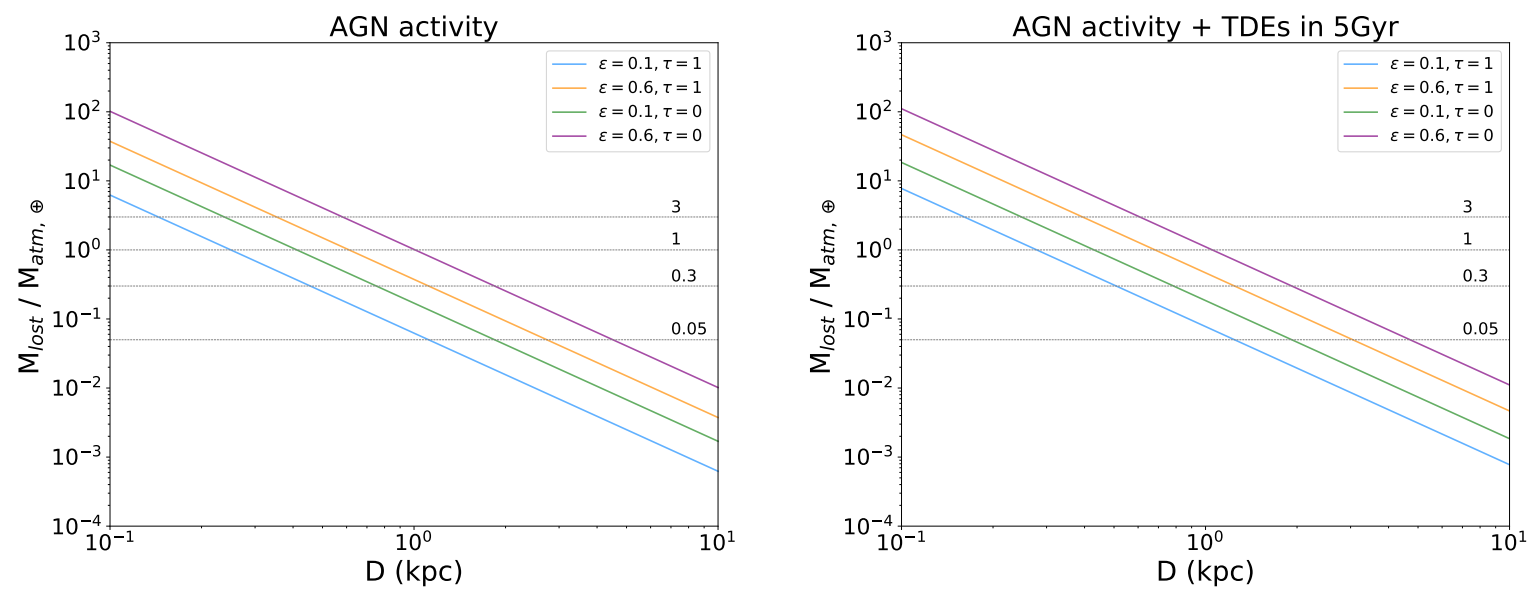

Figure 2. Total mass (in units of the Earth' $\breve{Z}$ Źs present-day atmospheric mass) lost by a terrestrial planet at distance $D$. The left panel depicts the contribution from the AGN phase (Balbi \& Tombesi 2017). The right panel illustrates the total radiative feedback from Sgr A*, i.e., considering the AGN phase and TDEs over a span of $5 \mathrm{Gyr}$. We show the results for two values of the efficiency of hydrodynamic escape $(\varepsilon)$ and the optical depth $(\tau)$, which parametrizes the presence $(\tau=1)$ or absence $(\tau=0)$ of the obscuring torus during the AGN phase.

extinction (Wei et al. 2014), suggesting that similar detrimental effects are conceivable on other worlds.

\subsection{Biological damage}

We begin with estimating $D_{c}$ by employing (10) for the two different values of $\mathcal{F}_{c}$ described in Sec. 2.2. After further simplification, we obtain $D_{c} \approx 3.42 \mathrm{kpc}$ and $D_{c} \approx 34.2 \mathrm{kpc}$ for prokaryotes and eukaryotes, respectively, after using the parameters for $\mathrm{Sgr} \mathrm{A}^{*}$ and choosing $\tau=0$. Therefore, only at distances $D>D_{c}$ would these organisms not be susceptible to major damage. In comparison, Balbi \& Tombesi (2017) carried out analogous calculations for the AGN phase of Sgr A*, and obtained $D_{c} \approx 1.33 \mathrm{kpc}$ and $D_{c} \approx 13.3 \mathrm{kpc}$ for prokaryotes and eukaryotes, respectively. Hence, insofar as $D_{c}$ is concerned, it would appear as though TDEs have a slightly more severe impact than the AGN phase.

At first glimpse, this appears to contradict the indubitable existence of complex muticellularity on Earth over the past $>1 \mathrm{Gyr}$ (Knoll 2011), because the Sun is $8 \mathrm{kpc}$ from the Galactic center, which is smaller than $D_{c}$. However, this is where the two caveats delineated in Sec. 2.2 come into play. To begin with, selecting $D=8$ $\mathrm{kpc}$ in (8) yields a fluence that is more than an order of magnitude smaller than $\mathcal{F}_{\oplus}$, i.e., the solar XUV fluence in the same period. Moreover, the Earth's atmosphere would prevent the bulk of this XUV radiation from penetrating to the surface. Hence, we reiterate that the above two estimates for $D_{c}$ are upper bounds, and apply to worlds with very tenuous atmospheres.

The next factor that we consider is the biological damage via UV-C radiation incident on an Earth-analog, following the procedure described after (11). After selecting $\tau=0$ and the parameters for $\mathrm{Sgr} \mathrm{A}^{*}$, we obtain a critical distance of $0.6 \mathrm{pc}$ if we suppose that $L$ is the average luminosity of the TDE in (11), and $1.7 \mathrm{pc}$ if we use the peak luminosity instead. In comparison, the corresponding critical distance for $\mathrm{Sgr} \mathrm{A}^{*}$ during the active phase is $9.4 \mathrm{pc}$ (Lingam et al. 2019), implying that the zones of biological damage associated with TDEs and AGNs are typically within an order of magnitude of each other.

However, the above distances are merely loose lower bounds on the zones of biological damage. At such close distances, the XUV fluence is many orders of magnitude higher than that from a Sunlike star, and will therefore have potentially severe consequences for biota, even when the majority is absorbed by the atmosphere. There is also the issue of atmospheric evaporation as noted in Sec. 3.1; only Super-Earths with massive atmospheres would avoid total atmospheric loss at these distances (Chen et al. 2018); such worlds are quite common in the Solar neighborhood (Kaltenegger 2017). Lastly, at such distances, planets are subjected to regular impacts by relativistic gas and dust that could pose additional impediments for habitability (Sturm et al. 2018).

\section{CONCLUSION}

In this paper, we analyzed the impact of TDEs on galactic habitability by taking two major factors dependent on high-energy radiation into consideration: atmospheric loss and biological damage. We chose to focus on $\mathrm{Sgr} \mathrm{A}^{*}$ because its fundamental parameters are well constrained, and due to the fact that we know of at least one planet hosting life (i.e., Earth) in the Galaxy.

In the context of atmospheric depletion, we employed a simple hydrodynamic model to model this effect. We concluded that planets with Earth-like atmospheres may undergo total depletion over a span of 5 Gyr up to distances of $D \sim 0.1-1 \mathrm{kpc}$; the variation in the distance arises due to the free parameters involved. Next, we investigated the constraints on biological damage arising from TDEs. Although our model suggests that significant biological hazards are posed by the TDEs up to distances of $D \sim 10 \mathrm{kpc}$, this constitutes a loose upper bound in all likelihood. At distances of $D \sim 0.1-1 \mathrm{kpc}$, we found that the XUV fluences are higher than, or comparable to, those received by temperate planets around Sun-like stars, implying that worlds within this zone might experience non-trivial biological damage because of the higher radiation levels.

As per our findings, worlds at distances of $D \lesssim 0.1-1 \mathrm{kpc}$ may be susceptible to major perturbations of their biosphere by TDEs. Quite intriguingly, the mean interval between these disruptions is $\gtrsim 10^{4}$ yrs (Komossa 2015; Stone et al. 2020), which is not far removed from the characteristic timescales of $\sim 10^{4}-10^{5} \mathrm{yrs}$ 
associated with Milankovitch cycles (Berger 1988). Therefore, for such worlds, it is conceivable that TDEs could potentially play the role of Milankovitch cycles in regulating climactic and biodiversity patterns. At even closer distances to the Galactic center, TDEs might initiate periodic mass extinctions, along the lines of Earth's alleged extinction periodicity of order 10 Myr (Bambach 2006). Whether life can persist over long timescales on this class of worlds would depend on the recovery timescale after extinction(s), which in turn is partly governed by the severity of the extinction(s); for instance, the most severe mass extinction in the Phanerozoic, in the end-Permian, entailed a recovery time of $\sim 10 \mathrm{Myr}$ (Chen \& Benton 2012).

To summarize, two broad conclusions emerge from this work. First, the cumulative deleterious impact of TDEs on habitability is broadly comparable to that of AGNs. Second, as the distance up to which the effects on surficial habitability are prominent could be $\sim 0.1-1 \mathrm{kpc}$ from the central black hole of the Milky Way, some fraction of the total number of planetary systems in the Milky Way within this region may have been adversely affected by the combined action of TDEs and the active phase of our Galaxy. ${ }^{3}$ Although there are some vital factors that have been set aside, our analysis suggests that TDEs might exert a substantive influence on planetary habitability at distances $\lesssim 0.1-1 \mathrm{kpc}$ from the central black hole of the MW. Hence, not only may they merit further investigation along the same lines as supernovae, gamma-ray bursts and AGNs, but they ought to be incorporated into state-of-the-art numerical models that track the spatio-temporal evolution of of galactic habitability at subkpc distances (Dayal et al. 2015; Forgan et al. 2017; Stojković et al. 2019).

\section{ACKNOWLEDGEMENTS}

A.B. acknowledges support by the Italian Space Agency (ASI, DCVUM-2017-034, grant number 2019-3 U.O Life in Space) and by grant number FQXi-MGA-1801 and FQXi-MGB-1924 from the Foundational Questions Institute and Fetzer Franklin Fund, a donor advised fund of Silicon Valley Community Foundation.

\section{DATA AVAILABILITY}

The data underlying this article are available in the article.

\section{REFERENCES}

Alexander T., 2017, ARA\&A, 55, 17

Amaro-Seoane P., Chen X., 2019, J. Cosmology Astropart. Phys., 2019, 056 Arney G., et al., 2016, Astrobiology, 16, 873

Auchettl K., Guillochon J., Ramirez-Ruiz E., 2017, ApJ, 838, 149

Balbi A., Tombesi F., 2017, NatSR, 7, 16626

Bambach R. K., 2006, AREPS, 34, 127

Berger A., 1988, RvGeo, 26, 624

Brain D. A., Bagenal F., Ma Y. J., Nilsson H., Stenberg Wieser G., 2016, JGRE, 121, 2364

Branch D., Wheeler J. C., 2017, Supernova Explosions. Springer-Verlag, doi:10.1007/978-3-662-55054-0

\footnotetext{
${ }^{3}$ Note that these distances are applicable only in the context of surfacebased habitability. The number of worlds with subsurface oceans (and potentially with biospheres) might outnumber those with surface liquid water by a few orders of magnitude (Lingam \& Loeb 2019a).
}

Catling D. C., Kasting J. F., 2017, Atmospheric Evolution on Inhabited and Lifeless Worlds. Cambridge University Press

Chen Z.-Q., Benton M. J., 2012, NatGe, 5, 375

Chen H., Forbes J. C., Loeb A., 2018, ApJ, 855, L1

Clarke J. N., 1981, Icarus, 46, 94

Cleaves H. J., Miller S. L., 1998, PNAS, 95, 7260

Cockell C. S., Knowland J., 1999, Biol. Rev., 74, 311

Cockell C. S., et al., 2016, AsBio, 16, 89

Dai L., McKinney J. C., Roth N., Ramirez-Ruiz E., Miller M. C., 2018, ApJ, 859, L20

Dartnell L. R., 2011, AsBio, 11, 551

Dayal P., Cockell C., Rice K., Mazumdar A., 2015, ApJ, 810, L2

Dong C., Lingam M., Ma Y., Cohen O., 2017, ApJ, 837, L26

Dong C., Jin M., Lingam M., Airapetian V. S., Ma Y., van der Holst B., 2018, PNAS, 115, 260

Ellis J., Schramm D. N., 1995, PNAS, 92, 235

Forbes J. C., Loeb A., 2018, MNRAS, 479, 171

Forgan D., Dayal P., Cockell C., Libeskind N., 2017, IJAsB, 16, 60

Frank J., Rees M. J., 1976, MNRAS, 176, 633

French K. D., Wevers T., Law-Smith J., Graur O., Zabludoff A. I., 2020, SSRv, 216, 32

Gehrels N., Laird C. M., Jackman C. H., Cannizzo J. K., Mattson B. J., Chen W., 2003, ApJ, 585, 1169

Gonzalez G., 2005, OLEB, 35, 555

Gowanlock M. G., Morrison I. S., 2018, The Habitability of our Evolving Galaxy. Academic Press, pp 149-171, doi:10.1016/B978-0-12-8119402.00007-1

Hills J. G., 1975, Nature, 254, 295

Kaib N. A., 2018, Galactic Effects on Habitability. Springer, pp 3091-3109, doi:10.1007/978-3-319-55333-7_59

Kaltenegger L., 2017, ARA\&A, 55, 433

Knoll A. H., 2011, AREPS, 39, 217

Komossa S., 2015, JHEAp, 7, 148

Krolik J. H., 1999, Active Galactic Nuclei: From the Central Black Hole to the Galactic Environment. Princeton University Press

Lacy J. H., Townes C. H., Hollenbach D. J., 1982, ApJ, 262, 120

Lingam M., Loeb A., 2019a, IJAsB, 18, 112

Lingam M., Loeb A., 2019b, RvMP, 91, 021002

Lingam M., Ginsburg I., Bialy S., 2019, ApJ, 877, 62

Liu C., Chen X., Du F., 2020, ApJ, p. arXiv:2002.03086

Lodato G., Franchini A., Bonnerot C., Rossi E. M., 2015, JHEAp, 7, 158

Lu W., Kumar P., 2018, ApJ, 865, 128

Melott A. L., Thomas B. C., 2011, Astrobiology, 11, 343

Owen J. E., 2019, AREPS, 47, 67

Padmanabhan H., Loeb A., 2020, arXiv e-prints, p. arXiv:2003.07365

Prantzos N., 2008, Space Sci. Rev., 135, 313

Rees M. J., 1988, Nature, 333, 523

Ribas I., Guinan E. F., Güdel M., Audard M., 2005, ApJ, 622, 680

Rugheimer S., Segura A., Kaltenegger L., Sasselov D., 2015, ApJ, 806, 137

Saxton R. D., Read A. M., Komossa S., Lira P., Alexander K. D., Wieringa M. H., 2017, A\&A, 598, A29

Scalo J., Wheeler J. C., 2002, ApJ, 566, 723

Stojković N., Vukotić B., Martinović N., Ćirković M. M., Micic M., 2019, MNRAS, 490, 408

Stone N. C., Kesden M., Cheng R. M., van Velzen S., 2019, GReGr, 51, 30

Stone N. C., Vasiliev E., Kesden M., Rossi E. M., Perets H. B., AmaroSeoane P., 2020, Space Sci. Rev., 216, 35

Sturm E., et al., 2018, Nature, 563, 657

Urry C. M., Padovani P., 1995, PASP, 107, 803

Wei Y., et al., 2014, E\&PSL, 394, 94

Wisłocka A. M., Kovačević A. B., Balbi A., 2019, A\&A, 624, A71

This paper has been typeset from a $\mathrm{T}_{\mathrm{E}} \mathrm{X} / \mathrm{L} \mathrm{T} \mathrm{T}_{\mathrm{E}} \mathrm{X}$ file prepared by the author. 